\title{
Transcranial optical imaging reveals a pathway for optimizing the delivery of immunotherapeutics to the brain
}

\author{
Benjamin A. Plog, ${ }^{1,2}$ Humberto Mestre, ${ }^{1,3}$ Genaro E. Olveda, ${ }^{1}$ Amanda M. Sweeney, ${ }^{1}$ H. Mark Kenney, \\ Alexander Cove, ${ }^{1}$ Kosha Y. Dholakia, ${ }^{1}$ Jeffrey Tithof, ${ }^{4}$ Thomas D. Nevins, ${ }^{5}$ Iben Lundgaard, ${ }^{1}$ \\ Ting Du, ${ }^{1,6}$ Douglas H. Kelley, ${ }^{4}$ and Maiken Nedergaard ${ }^{1,3}$ \\ ${ }^{1}$ Center for Translational Neuromedicine, Department of Neurosurgery, ${ }^{2}$ Department of Pathology, and \\ ${ }^{3}$ Department of Neuroscience, University of Rochester Medical Center, Rochester, New York, USA. \\ ${ }^{4}$ Department of Mechanical Engineering and ${ }^{5}$ Department of Physics and Astronomy, University of Rochester, Rochester, \\ New York, USA. '́Laboratory of Metabolic Brain Diseases, Institute of Metabolic Disease Research and Drug Development, \\ China Medical University, Shenyang, China.
}

Despite the initial promise of immunotherapy for CNS disease, multiple recent clinical trials have failed. This may be due in part to characteristically low penetration of antibodies to cerebrospinal fluid (CSF) and brain parenchyma, resulting in poor target engagement. We here utilized transcranial macroscopic imaging to noninvasively evaluate in vivo delivery pathways of CSF fluorescent tracers. Tracers in CSF proved to be distributed through a brain-wide network of periarterial spaces, previously denoted as the glymphatic system. CSF tracer entry was enhanced approximately 3 -fold by increasing plasma osmolality without disruption of the blood-brain barrier. Further, plasma hyperosmolality overrode the inhibition of glymphatic transport that characterizes the awake state and reversed glymphatic suppression in a mouse model of Alzheimer's disease. Plasma hyperosmolality enhanced the delivery of an amyloid- $\beta$ (A $\beta$ ) antibody, obtaining a 5 -fold increase in antibody binding to $A \beta$ plaques. Thus, manipulation of glymphatic activity may represent a novel strategy for improving penetration of therapeutic antibodies to the CNS.

Authorship note: BAP and HM contributed equally to this work.

Conflict of interest: The authors have declared that no conflict of interest exists.

License: Copyright 2018, American Society for Clinical Investigation.

Submitted: March 6, 2018 Accepted: September 6, 2018 Published: October 18, 2018

\section{Reference information:} JCI Insight. 2018;3(20): e120922. https://doi.org/10.1172/jci. insight.120922.

\section{Introduction}

Therapies based on monoclonal antibodies (mAbs) are currently being developed for CNS diseases, such as Alzheimer's disease (AD) (1), Parkinson's disease (2), amyotrophic lateral sclerosis (3), frontotemporal lobar dementia (4), and CNS tumors (5). Perhaps the most notable application of $\mathrm{mAb}$ is seen in efforts to enhance amyloid- $\beta(\mathrm{A} \beta)$ clearance from brain $(4,6,7)$. Despite promising preclinical results with this approach, results of clinical trials have been unimpressive and plagued by adverse events (8-10). This may reflect the poor penetration of therapeutic mAbs to brain, resulting in inadequate target engagement (11). Failure of anti-A $\beta$ immunotherapies to engage with plaques located in deep brain structures may contribute to the lack of translation of these therapies into routine $\mathrm{AD}$ treatment $(4,12)$. Due to the invasiveness and higher degree of complications associated with injections directly to cerebrospinal fluid (CSF), therapeutic antibodies are most commonly administered by intravenous infusion (13-15) However, circulating antibodies have low penetration of the blood-brain barrier (BBB), with only $0.1 \%$ $0.2 \%$ entering brain (16). As a result, therapeutic antibodies are often given at doses 1,000 -fold greater than the concentration needed to achieve adequate binding of the target antigen in peripheral tissues $(1,17)$, and these high doses in clinical trials increase the prevalence of adverse events $(1,9,18)$ such as amyloid-related imaging abnormalities (ARIA) $(19,20)$. While the human mAb, aducanumab, induced dose-dependent reduction in soluble and insoluble $\mathrm{A} \beta$ and slowed clinical decline in prodromal or mild $\mathrm{AD}$ patients, ARIA emerged as a major issue, limiting intravenous dosing (1). Conversely, a preclinical study showed that prolonged delivery of another anti-A $\beta$ mAb directly to the CSF reduces the antibody dosage required to observe a clinical benefit by at least 10 -fold, while enhancing clearance of cerebral amyloid angiopathy and preventing microhemorrhages (19). These findings have drawn renewed interest in optimizing CSF-based delivery of clinically promising therapeutic mAbs. 
Prior studies evaluating the transport of CSF macromolecules into the brain have revealed a bulk flow pathway along the perivascular space (PVS) surrounding the pial and penetrating arteries for CSF circulation into the brain (21-25). Although antibodies show limited diffusive transport in the CNS extracellular space $(26,27)$, harnessing perivascular and parenchymal convective flows could potentially enhance their delivery into the brain. This bulk flow pathway, termed the glymphatic system for its role in solute clearance and its dependence on the glial water channel aquaporin-4 (AQP4) (21), represents an ideal mechanism for drug delivery to the CNS. The fast, convective fluid flow within the glymphatic system effectively delivers solutes of high-molecular weight (21) but is strongly regulated by brain state (28), aging (29), arterial pulsatility (30), and body posture (31). We here describe what we believe to be a novel noninvasive transcranial macroscopic imaging approach that allows us to track cortical CSF flow in real time in the intact brain of living mice. We used this technique to evaluate whether therapeutic enhancement of glymphatic influx would increase the delivery of CSF-based tracers into the brain. Based on earlier results (32-34), we further tested whether increasing plasma osmolality with hypertonic saline (HTS) or mannitol and, thereby, decreasing intracranial pressure (ICP) would increase glymphatic influx without disruption of the BBB.

\section{Results}

In vivo transcranial fluorescence macroscopy allows noninvasive brain-wide imaging of glymphatic flow and confirms 2-photon and ex vivo microscopic findings of reduced CSF influx in awake and Aqp4-1- mice. Prior studies of CSF interstitial fluid (ISF) exchange within the glymphatic system have utilized ex vivo conventional fluorescence microscopy and in vivo 2-photon microscopy to image CSF- or ISF-based tracer fluxes (21, 22, 28, $29,35,36)$. While superior for evaluation of brain-wide glymphatic function, including its detailed cellular and molecular organization, ex vivo imaging of brain sections lacks dynamic temporal information, requiring that indirect inferences be made about temporal patterns and rates of glymphatic flow. Conversely, in vivo 2-photon imaging permits real-time determination of rates of CSF tracer appearance in cerebral tissues in individual mice, albeit with a relatively narrow field of view and shallow imaging depth $(21,28,29)$.

Consequently, we developed a technique for noninvasive in vivo time-lapse imaging of transcranial glymphatic flows using fluorescence macroscopy (Supplemental Figure 1; supplemental material available online with this article; https://doi.org/10.1172/jci.insight.120922DS1). The technique consists of imaging fluorescent tracers delivered into the cisterna magna of live rodents through an intact skull using an LED illumination source for fluorophore excitation and a macro zoom microscope with high-efficiency CMOS camera for fluorescence detection. The tunable LED can achieve fast switching between wavelengths, and quad filter cubes allow for high-speed, multichannel image acquisition. The macroscope has a wide field of view, enabling mesoscopic imaging of the entire cortical surface and a penetration depth of up to 1-2 $\mathrm{mm}$ (Supplemental Figure 2). In validating this technique, we first replicated prior in vivo and ex vivo findings of reduced glymphatic CSF influx in awake mice and mice lacking AQP4 $\left(A q p 4^{-/}\right)(21,28)$. Anesthetized mice in all groups had reflection of the scalp overlying the dorsal calvarium and cannula placement within the cisterna magna. Groups of wild-type and $A q p 4^{-/-}$mice were subsequently maintained under ketamine/xylazine (KX) anesthesia ( $\mathrm{KX}$ and $\mathrm{KX}-A q p 4^{--}$), while a separate group of wild-type mice had a metallic plate secured to the skull for head immobilization and were then placed in a plastic restraint tube prior to waking up (Awake). Subsequently, mice were moved to the stage of a fluorescence macroscope for dynamic image acquisition, Alexa Fluor 647-conjugated BSA (BSA-647, $66 \mathrm{kDa}$ ) was injected into the cisterna magna (Figure 1, A and B). Intracisternal infusion caused a mild, transient increase in ICP that normalized before the appearance of tracer (30). Fluorescence was detectable as soon as the tracers arrived in the basal cistern, approximately 5-6 mm below the cortical surface (Supplemental Figure 3). The influx of BSA-647 into the brain was imaged over 30 minutes before brain harvesting, fixation in 4\% paraformaldehyde (PFA), and washing in buffer (Figure 1B). In both anesthetized and awake mice, this transcranial macroscopic approach revealed a pattern of glymphatic CSF influx identical to that previously characterized using in vivo 2-photon (Supplemental Figure 4) and ex vivo imaging techniques. The fluorescent protein tracer first appeared within the large rostral and caudal subarachnoid spaces, such as the olfacto-frontal cistern and pineal recess, and was carried within minutes over the dorsal cerebral convexity within pial periarterial spaces (Supplemental Figure 4); this topography followed the territories of the anterior and posterior cortical segments of the middle cerebral artery (MCA; Supplemental Figures 4 and 5). We note that the infusion pump was stopped at 5 minutes or when faint fluorescent signal was first noted at the base of the MCA, indicating that all subsequent tracer appearance could be attributed to physiologic bulk flow. Toward the end of the 30-minute 
A

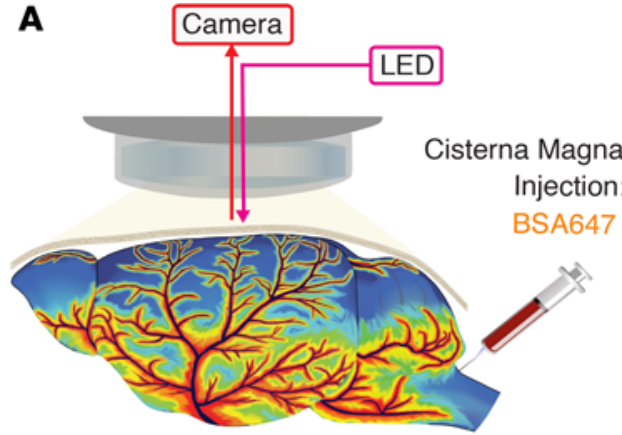

B

\begin{tabular}{|c|c|} 
Injection & Fixation \\
\hline $5 \mathrm{~min}$ & $25 \mathrm{~min}$ \\
\hline
\end{tabular}

C

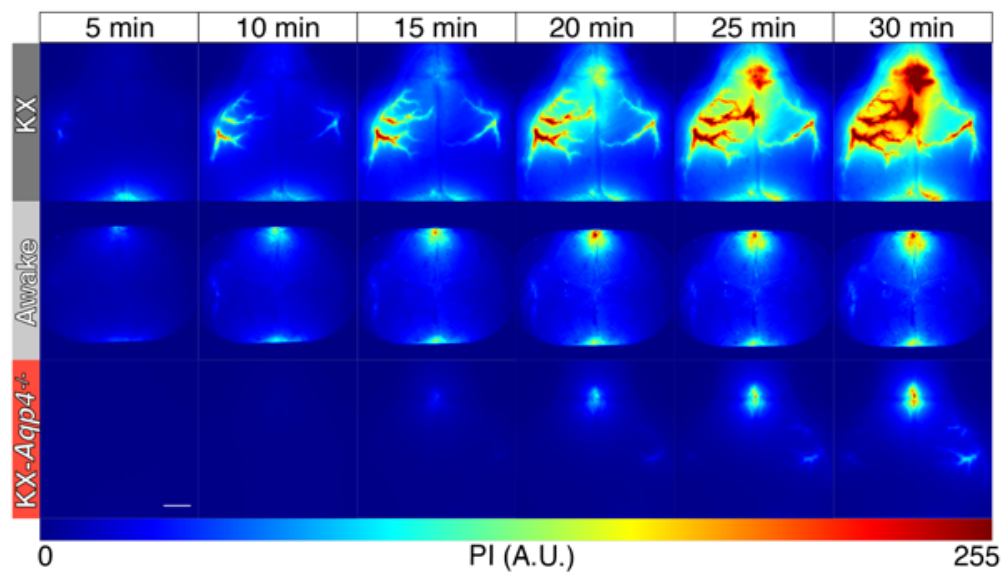

D

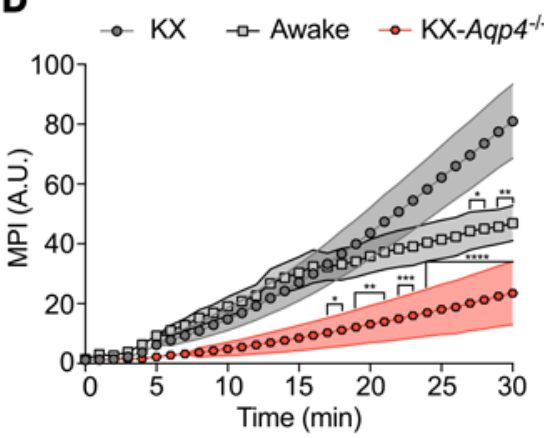

E

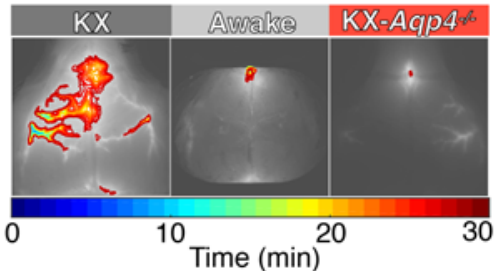

G

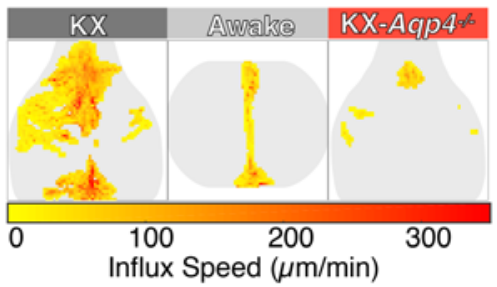

$\mathbf{F} \rightarrow \mathrm{KX} \quad \rightarrow$ Awake $\rightarrow \mathrm{KX}-\mathrm{Aqp} 4^{-1}$

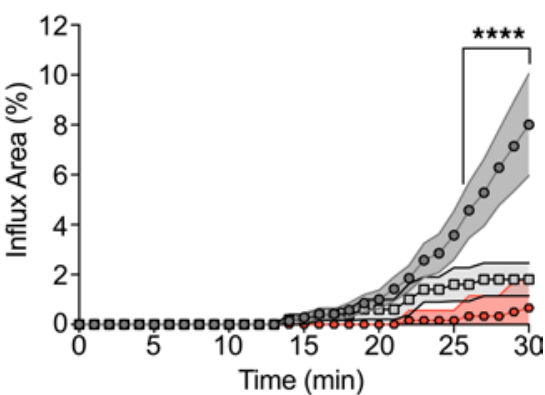

H
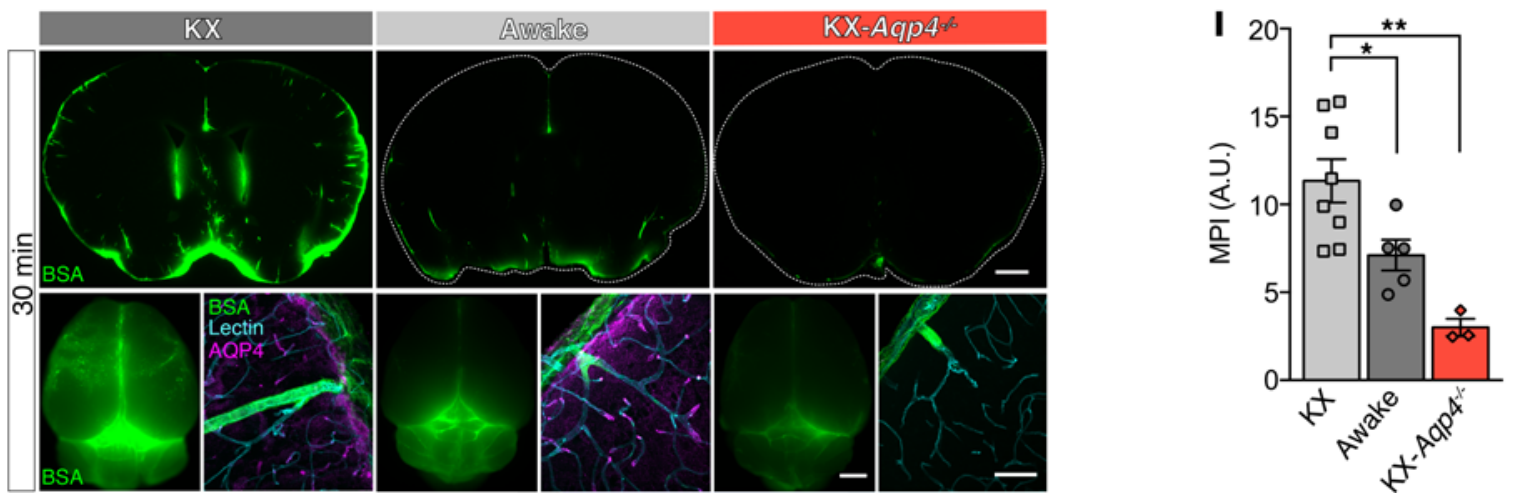

Figure 1. In vivo transcranial brain-wide imaging of CSF influx. (A). Mice were imaged through an intact skull using a macroscope. (B) A fluorescent protein tracer (BSA-647 nm) was delivered into the cisterna magna ( $2 \mu \mathrm{l} / \mathrm{min}, 5$ minutes) and tracer influx was imaged for 30 minutes from the start of the injection. All mice received i.p. isotonic saline at the onset of the intracisternal injection. (C) Representative time-lapse images of CSF influx

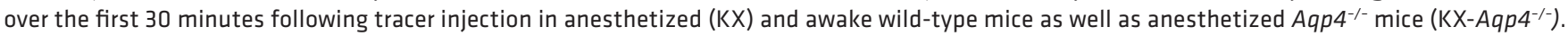
Images (8-bit pixel depth) are color coded to depict pixel intensity (PI) in arbitrary units (AU). Scale bar: 2 mm. Fluorescence was detected as early as 5 minutes after infusion at the base of the brain approximately 5-6 mm below the dorsal cortical surface. (D) Quantification of mean pixel intensity (MPI) for the 30-minute in vivo imaging series depicted in C (mean \pm SEM; $n=5-7$ mice/group; repeated-measures 2-way ANOVA, Sidak's multiple comparisons test; ${ }^{*} P<0.05$, ${ }^{* *} P<0.01$, ${ }^{* *} P<0.001$, ${ }^{* * * *} P<0.0001 \mathrm{vs}$. $\mathrm{KX}$ ). (E) Representative front-tracking analysis of CSF tracer influx over the imaging session. Fronts are time coded in minutes. (F) Quantification of the influx area over time from analysis E (mean \pm SEM; $n=5-7$ mice/group; repeated-measures 2-way ANOVA, Sidak's multiple comparisons test; ${ }^{* * *} P<0.0001 \mathrm{KX}$ vs. awake and KX- Aqp4 $4^{-/}$). (G) Average influx speed maps $(\mu \mathrm{m} / \mathrm{min})$ generated from group data in $\mathbf{C}$ and $\mathbf{E}$. (H) Representative conventional fluorescence images of brains ex vivo upon removal from the cranium (whole brains; scale bar: $2 \mathrm{~mm}$ ) and after coronal sectioning to evaluate tracer penetrance deep into the cortical surface (coronal sections; scale bar: $1 \mathrm{~mm}$ ) in the $\mathrm{KX}$ and awake wild-type and $\mathrm{KX}$-anesthetized Aqp4 $4^{-/}$groups. High-magnification images of perivascular tracer were acquired using laser scanning confocal microscopy (immunohistochemical staining; scale bar: $50 \mu \mathrm{m}$ ). (I) Quantification of ex vivo coronal section fluorescence MPI for the KX and awake wild-type and KX-anesthetized Aqp4 $4^{-/-}$groups (mean $\pm \mathrm{SEM} ; n=3-8$ mice/group; 1-way ANOVA, Tukey's multiple comparisons test; $\left.{ }^{*} P<0.05,{ }^{* *} P<0.01\right)$. 
imaging experiment, tracer started to accumulate within the PVS of cortical bridging veins adjacent to the dural sinuses. Confirming previously reported findings (21, 28, 37-39), there was significantly less glymphatic influx in both the awake and $\mathrm{KX}-A q p 4^{4^{-}-}$groups compared with that in the $\mathrm{KX}$ mice (Figure $1, \mathrm{C}$ and $\mathrm{D}$, and Supplemental Video 1). Interestingly, at the 30-minute time point, glymphatic influx was significantly lower in anesthetized Aqp $4^{-/}$mice than in the awake group (Figure 1D), suggesting that effect on glymphatic function due to deletion of water channels exceeded effects of state of consciousness. As described above, prior studies of CSF-based delivery of intrathecal solutes to brain could not simultaneously quantify the surface area covered by the tracer and influx kinetics. Even MRI, despite a unique ability to characterize spatial distribution and temporal dynamics, lacks the spatial resolution required to evaluate CSF flows at the level of the PVS (22). Using front-tracking analysis in combination with a macroscopic imaging paradigm to overcome this limitation (Supplemental Video 2), we could demonstrate that CSF flow within pial periarterial spaces was higher in KX anesthetized mice and occupied approximately $10 \%$ of the dorsal cortical surface compared with under $2 \%$ in the awake and $\mathrm{KX}-A q p 4^{--}$groups (Figure 1, E-G). Further, in the awake and knockout groups, PVSs were essentially devoid of tracer, with most of the fluorescent area confined to the olfacto-frontal cistern (Figure 1E). To exclude the possibility that the imaged glymphatic fluxes were occurring exclusively within the subarachnoid space, the harvested brains underwent conventional fluorescence imaging ex vivo. This analysis showed that the distribution of fluorescent signal throughout the dorsal cortex matched that seen at the terminal 30-minute time point of the sequence in vivo (Supplemental Figure 6), thus supporting that the CSF flows observed with the macroscope were occurring at the tissue level and not within the subarachnoid space. Following coronal sectioning, brain slices were imaged to determine the degree of tracer penetrance into deeper cerebral structures. Again, in parallel to observations during the in vivo imaging series, significantly less tracer was present in brain tissue of the awake and $\mathrm{KX}-A q p 4^{4^{--}}$mice than in the KX group (Figure 1H, top, and Figure 1I). Finally, high-resolution confocal images showed that PVS tracer distribution was higher in the KX cohort than in the awake and $\mathrm{KX}-A q p 4^{-1-}$ mice (Figure $1 \mathrm{H}$, bottom right), confirming the in vivo observation of absent perivascular influx within these groups and confirming that tracer influx does not occur via diffusion along the pial surface (27). Collectively, these findings validate the use of fluorescence macroscopy for brain-wide, transcranial imaging of in vivo glymphatic CSF influx and recapitulate prior work demonstrating the dependence of these flows on perivascular AQP4 expression and the enlargement of interstitial space volume that accompanies sleep (21, 28). Additionally, these data suggest that increased resistance to fluid flow between the perivascular and interstitial spaces due to AQP4 deletion has a much more profound suppressive effect on glymphatic flow than does wakefulness-related contraction of the interstitial space. The recent critique of the key role of AQP4 in glymphatic function (40) has been challenged by a recent study from 4 independent labs demonstrating an essential role of AQP4 in solute dispersion in the mouse brain (37).

Plasma hyperosmolality significantly increases CSF influx in anesthetized mice. While the role of the glymphatic pathway both in the clearance $(21,28,29,38,41,42)$ and delivery $(21,39,43-48)$ of solutes has been established by multiple groups, prior studies using fluorescent, radiolabeled, and gadolinium-based tracers also suggest that convective flow through this pathway might be exploited to deliver exogenous substances to targets deep within the brain parenchyma. Previous work has shown that the flow of CSF into the brain is increased in circumstances where the brain's volume regulatory capacity is challenged, as occurs with plasma hypertonicity (32). Thus, we asked whether plasma hyperosmolality could enhance the delivery of CSF-based tracers to a greater volume of brain and whether such enhancement occurs via the network of PVSs comprising the glymphatic pathway.

Plasma hypertonicity is often induced clinically for the treatment of elevated ICP using either HTS or mannitol infusions (49). In the experiments evaluating effects on glymphatic function, both hypertonicity methods were used to exclude specificity for HTS or mannitol. KX-anesthetized wild-type mice, prepared as above, received an i.p. injection of either isotonic saline (KX), HTS (+HTS), or hypertonic mannitol (+Mannitol) (Supplemental Figure 7, A and B), consistently resulting in significantly elevated plasma osmolality lasting 30 minutes (Supplemental Figure 7, C-E). Notably, at the plasma tonicities achieved in the HTS and mannitol-injected mice, there was no significant increase in BBB permeability (Supplemental Figure 8, A-C). In both treatment groups, immediately following i.p. injection of isotonic or hypertonic solutions, BSA-647 was injected to the cisterna magna, and the tracer's area of distribution and kinetics were imaged for 30 minutes using the transcranial microscopic approach described above (Figure $2 \mathrm{~A}$ ). In agreement with prior studies $(27,50,51)$, the main route of CSF tracer entry into brain was via the PVSs 
A

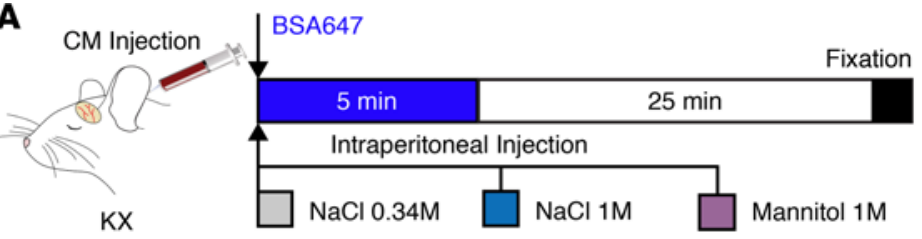

B

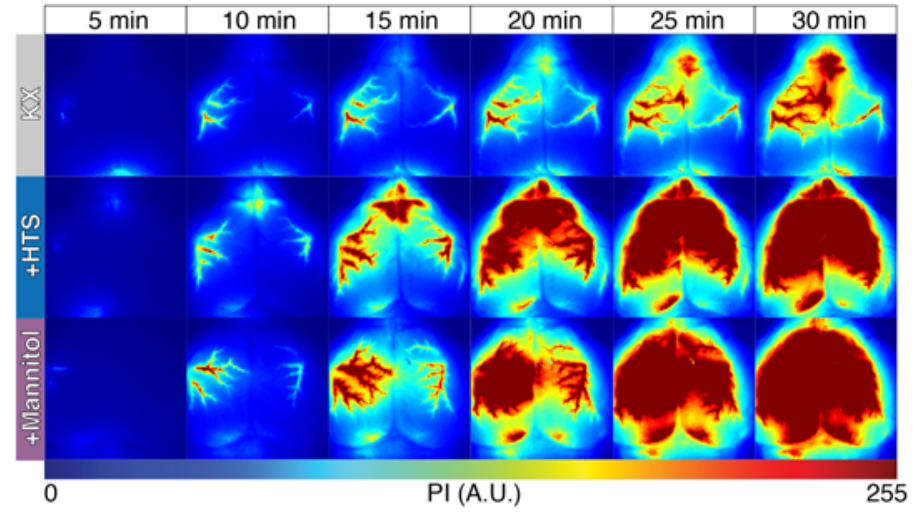

C

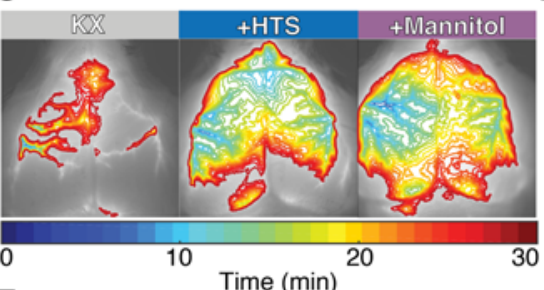

$\mathbf{E}$

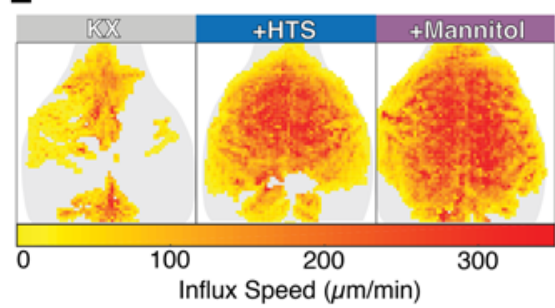

D $80, \cdot \mathrm{KX} *+\mathrm{HTS} *+$ Mannitol

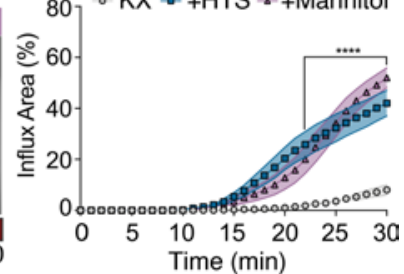

$\mathbf{F}$

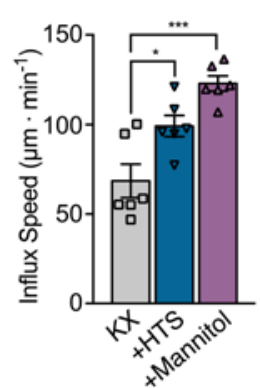

G
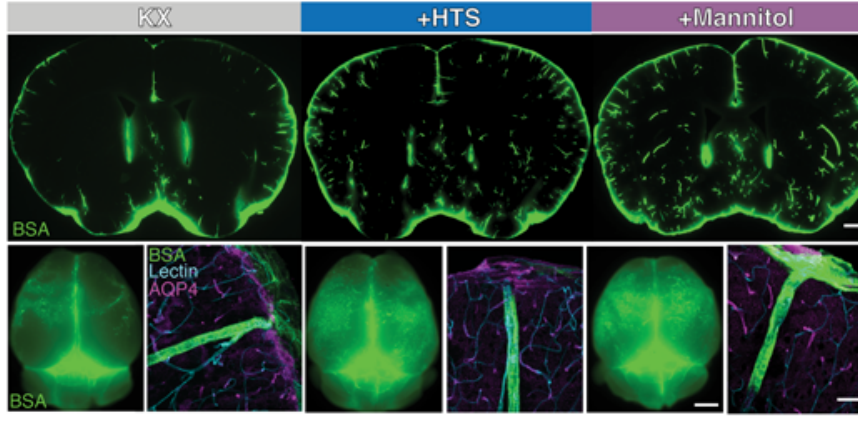

H

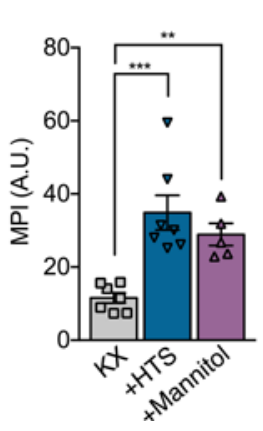

I

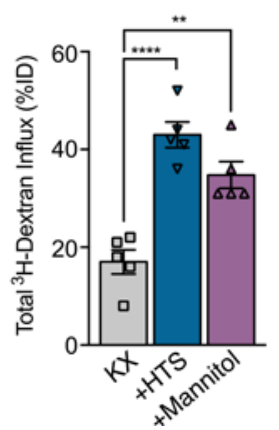

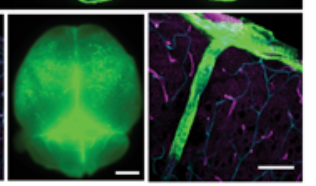

$\mathbf{J}$

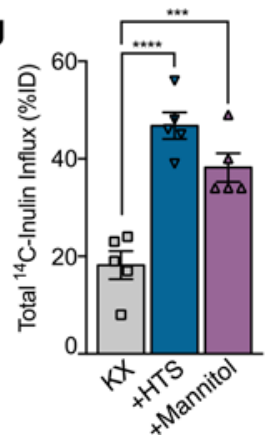

Figure 2. Plasma hypertonicity increases CSF influx in anesthetized mice. (A) Fluorescent BSA-647 was delivered into the cisterna magna (CM) of anesthetized mice. Mice received isotonic saline (KX), hypertonic saline (+HTS), or hypertonic mannitol (+Mannitol) i.p. at the onset of the CM injection. (B) Representative time-lapse images of BSA-647 influx over the immediate 30 minutes following CM injection in the $\mathrm{KX},+\mathrm{HTS}$, and +Mannitol groups. Images (8-bit pixel depth) are color coded to depict pixel intensity (PI) in arbitrary units (AU). Scale bar: $2 \mathrm{~mm}$. (C) Representative front-tracking analysis of CSF tracer influx over the imaging session for all groups. Fronts are time coded in minutes. (D) Quantification of the influx area over time (mean \pm SEM; $n=$ 6-7 mice/group; repeated-measures 2-way ANOVA, Sidak's multiple comparisons test; ${ }^{* * *} P<0.0001 \mathrm{KX}$ vs. +HTS and +Mannitol). (E) Tracer influx speed maps $(\mu \mathrm{m} / \mathrm{min})$ and $(\mathbf{F})$ quantification of mean influx speeds for all groups (mean \pm SEM; $n=6$ mice/ group; 1-way ANOVA, Tukey's multiple comparisons test; ${ }^{*} P<$ $\left.0.05,{ }^{* *} P=0.001\right)$. (G) Representative ex vivo conventional fluorescence images of intact brains upon removal from the cranium (scale bar: $2 \mathrm{~mm}$ ) and after coronal sectioning (scale bar: $1 \mathrm{~mm}$ ) from all groups. Coronal sections were imaged with high-powered confocal laser scanning microscopy to evaluate perivascular tracer (scale bar: $50 \mu \mathrm{m})$. (H) Quantification of ex vivo coronal section fluorescence MPI (mean \pm SEM; $n=5-7$ mice/group; 1-way ANOVA, Tukey's multiple comparisons test; ${ }^{*} P<0.01$, $\left.{ }^{* *} P=0.003\right)$. Total brain uptake of CSF-delivered (I) ${ }^{3} \mathrm{H}$-dextran $(40 \mathrm{kDa})$ or $(\mathrm{J})$ ${ }^{14} \mathrm{C}$-inulin $(6 \mathrm{kDa})$ in all 3 groups (mean $\pm \mathrm{SEM} ; n=5$ mice/group; 1-way ANOVA, Tukey's multiple comparisons test; ${ }^{* *} P=0.001$, $\left.{ }^{* *} P=0.0009,{ }^{* * *} P<0.0001\right)$ expressed as percentage injected dose (\%ID). The KX data set is the same that is used in Figure 1.

surrounding the MCA (Figure 2B and Supplemental Video 3). These data show that, in response to a volume regulatory challenge, the PVSs indeed act as fast conduits to deliver subarachnoid CSF into the volume-depleted brain. However, even more striking was the finding that plasma hypertonicity, due either to HTS or mannitol challenge, led to a nearly 5-fold increase in the influx area, with CSF tracer covering approximately $60 \%$ of the dorsal cortical surface, while reducing the time to delivery by roughly half (Figure 2, C and D). We saw significantly increased influx speeds over the entire cortical surface in the HTS and mannitol groups relative to the isotonic controls (Figure 2, E and F). This effect was independent of AQP4 expression, as plasma hypertonicity was able to override the inhibition of CSF influx seen in Aqp $4^{-1-}$ mice, to levels comparable with wild-types (Supplemental Figure 9). To rule out the possibility that this enhanced tracer influx was limited to the subarachnoid space, the brain and leptomeninges were removed from the calvarium, and cerebral tissues were washed prior to conventional ex vivo fluorescence microscopy. Here, the pattern of tracer distribution mirrored that seen at the 30 -minute time point of the in vivo imaging experiment, with most of the tracer occupying the subpial or pial PVSs (Figure 2G, bottom left, vs. Figure 2B, images under 30 minutes). Further, imaging of coronal brain sections showed significantly greater tracer signal at locations deep within the brain parenchyma of the HTS and mannitol groups, evidently having been carried into brain via the PVSs 
of cortical penetrating arteries (Figure 2G, top and bottom right, and Figure $2 \mathrm{H}$ ). Correlation analysis revealed a strong positive relationship between fluorescence signal captured using the newly described in vivo transcranial macroscopic approach $(R=0.914, P<0.0001)$, as well as ex vivo whole brain fluorescence $(R=0.921, P<0.0001)$, and the traditionally acquired signal from ex vivo coronal sections (Supplemental Figure 10, A and B), again suggesting that in vivo tracer dynamics are reflective of tissue level CSF and solute fluxes.

Finally, as the relationship between the amount of fluorescent tracer and relative fluorescence units is linear only at subsaturated signal levels, we used 2 radioisotope tracers, ${ }^{3} \mathrm{H}$-dextran $(40 \mathrm{kDa})$ and ${ }^{14} \mathrm{C}$-inulin $(6 \mathrm{kDa})$, to quantify hypertonicity-induced enhancement of glymphatic CSF influx to cerebral tissues. To confirm that entry of tracer within the brain parenchyma was not an artefact of the infusion paradigm but truly represented physiologic bulk flow, we injected the radioisotope tracers to the cisternal CSF at a rate and volume half that of the fluorescent tracers ( 1 vs. $2 \mu 1 / \mathrm{min}$, respectively over 5 minutes). We found a roughly $125 \%$ increase in the fractional tracer uptake to brain, with approximately $40 \%$ of the total injected tracer being delivered to the brain parenchyma in both conditions of plasma hyperosmolality (Figure 2, I and J). Notably, using plasma osmolality as the predictor, linear regression analysis of tissue radioisotope uptake versus in vivo transcranial or ex vivo coronal section fluorescence area showed significant positive relationships $(P<0.0001$ and $P=0.0014$, respectively), with similar regression slopes (Supplemental Figure 10, $\mathrm{C}$ and $\mathrm{D})$. These two independent lines of evidence support the concept that bulk flow mediates tracer influx irrespective of the more than 10-fold difference in molecular weight between fluorescent and radioisotope tracers and further suggests that noninvasively acquired in vivo fluorescence data and terminal radioisotope studies both offer similarly quantitative assessment of glymphatic dynamics.

Plasma hyperosmolality overrides arousal state-dependent inhibition of glymphatic function. As prior studies have demonstrated profound suppression of glymphatic function in conditions of arousal (28), we next asked whether plasma hypertonicity could overcome wakefulness-related inhibition of CSF influx. Using a similar approach as above, after i.p. injection of isotonic saline, HTS, or hypertonic mannitol, awake mice received an intracisternal injection of BSA-647 and underwent transcranial macroscopic imaging for a 30-minute period prior to brain removal and fixation (Figure 3A). Surprisingly, front-tracking analysis of the in vivo transcranial imaging sequence showed that plasma hyperosmolality evoked a circa 20-fold increase in tracer influx area at 30 minutes (Figure 3, B-D, and Supplemental Video 4). Further, tracer influx rates were roughly 1.5 -fold faster across the entire dorsal cortex in the awake hyperosmolar challenge groups (Figure 3, E and F). Finally, inspection of coronal sections showed significantly increased tracer influx to deep cerebral structures in both the HTS and mannitol-injected groups, matching observations in the KX-anesthetized cohort. Again, this enhanced tracer influx tended to occur via the PVSs of penetrating arteries (Figure 3, G and $\mathrm{H}$ ).

Hyperosmolar agents, such as HTS and mannitol, have been shown previously to influence mean arterial blood pressure (MAP), cerebral blood flow, and ICP $(52,53)$. Consequently, we next sought to determine if tonicity-induced changes in one of these parameters might be responsible for the observed enhancement of glymphatic CSF influx. Here, we found that both hyperosmolar challenges reduced MAP, but the effect of HTS was transient, resolving within 15-20 minutes of i.p. injection (Supplemental Figure 11A). Similarly, while there was a slight reduction in relative cerebral blood flow (rCBF) following mannitol administration, $\mathrm{rCBF}$ was preserved throughout the duration of the 30-minute recording in the HTS group (Supplemental Figure 11B). On the contrary, ICP significantly and consistently declined in both hyperosmolar groups relative to the isotonic controls (Supplemental Figure 11C). This net negative ICP resulted from the outflow of ISF across the BBB (Supplemental Figure 11D) and likely provided the necessary driving force to increase CSF influx to cerebral tissues. We emphasize that this transfer of brain water to the vascular column occurred across an intact BBB (Supplemental Figure 8, A-C).

Plasma hyperosmolality rescues glymphatic transport in 6-month-old APP/PS1 mice and enhances delivery and target engagement of an anti-A $\beta$ antibody. Having demonstrated in conditions of both anesthesia and wakefulness that plasma hyperosmolality increases CSF influx to brain, we next sought to determine if this paradigm could be used as a tool to improve brain-wide distribution of experimental therapeutics via the glymphatic pathway. Consequently, we asked whether plasma hypertonicity could rescue impairment of glymphatic CSF influx in a murine transgenic model of $\mathrm{AD}$ (APP/PS1 $1^{+/}$) and further whether an enhancement in glymphatic function could improve brain-wide delivery of an anti-A $\beta$ antibody and its interaction with both perivascular and parenchymal $\mathrm{A} \beta$ plaques. Using a similar approach as above, $\mathrm{KX}$ 
A

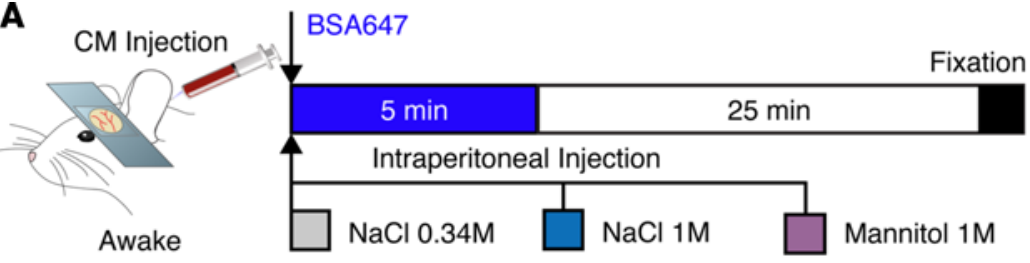

B

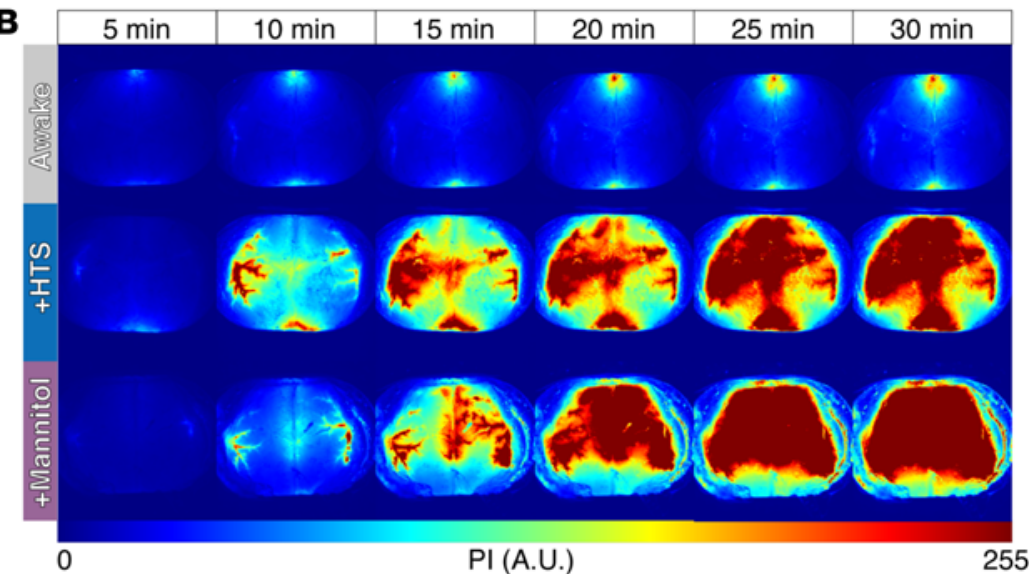

C

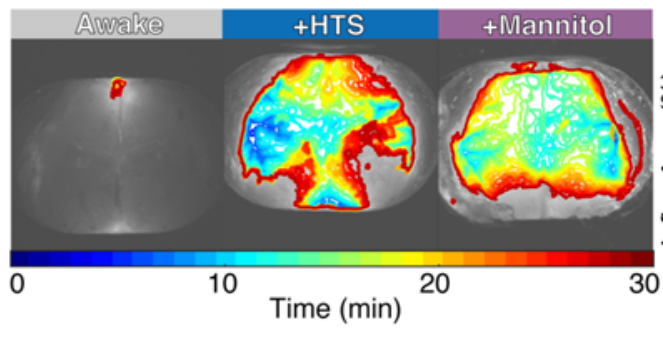

$\mathbf{E}$

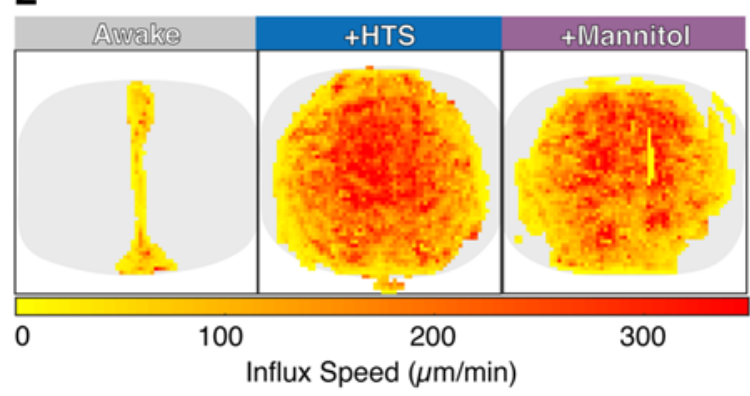

G

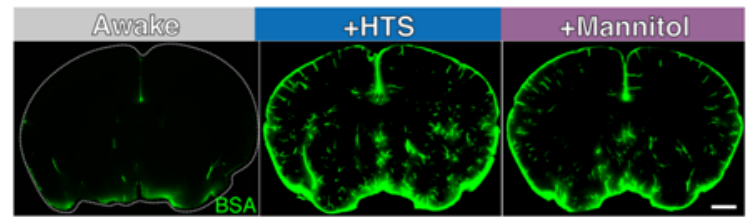

D

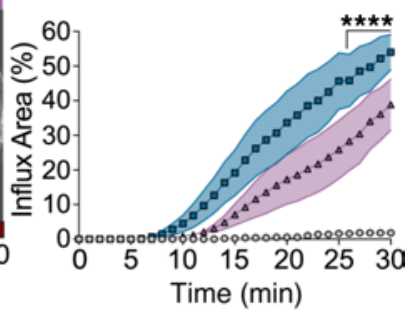

$\mathbf{F}$

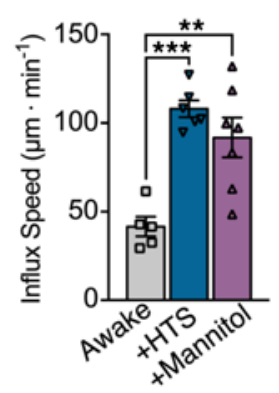

H

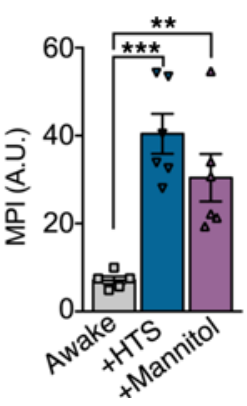

Figure 3. Plasma hypertonicity overrides arousal state inhibition of glymphatic function. (A) Head-plated, awake mice received intracisternal BSA-647. Mice received isotonic saline (Awake), hypertonic saline (+HTS), or hypertonic mannitol (+Mannitol) i.p. at the onset of the cisterna magna (CM) injection. (B) Representative time-lapse images of BSA-647 influx over the immediate 30 minutes following CM injection in the awake, +HTS, and +Mannitol groups. Images (8-bit pixel depth) are color coded to depict pixel intensity (PI) in arbitrary units (AU). Scale bar: $2 \mathrm{~mm}$. Fluorescence was first detected at the base of the brain approximately 5-6 $\mathrm{mm}$ below the dorsal cortical surface. (C) Representative front-tracking analysis of CSF tracer influx over the imaging session for all groups. Fronts are time coded in minutes. (D) Quantification of the influx area over time (mean \pm SEM; $n=5-7$ mice/group; repeated-measures 2-way ANOVA, Sidak's multiple comparisons test; ${ }^{* * *} P$ $<0.0001$ Awake vs. +HTS and +Mannitol). (E) Tracer influx speed maps $(\mu \mathrm{m} / \mathrm{min})$ and $(\mathbf{F})$ quantification of mean influx speeds for all groups (mean \pm SEM; $n=5-7$ mice/group; 1-way ANOVA, Tukey's multiple comparisons test; ${ }^{* *} P=0.0024,{ }^{* *} P=0.0003$ ). (C) Representative ex vivo coronal sections from all groups (scale bar: $1 \mathrm{~mm}$ ). (H) Quantification of ex vivo coronal section fluorescence MPI (mean \pm SEM; $n=5-6$ mice/group 1-way ANOVA, Tukey's multiple comparisons test; ${ }^{* *} P=$ $\left.0.0063,{ }^{* *} P=0.003\right)$. The Awake data set is the same that is used in Figure 1.

anesthetized 6-month-old APP/PS1 ${ }^{+/-}$mice received an i.p. injection of either isotonic saline (control) or + HTS immediately prior to intracisternal delivery of an Alexa Fluor 488-conjugated anti-A $\beta$ antibody (clone 6E10), which circulated for 120 minutes prior to brain removal and fixation (Figure 4, A and B). One day prior to intracisternal antibody injection, we obtained intravital labeling of $A \beta$ plaques with i.p. methoxy-X04 (MeX04) (Figure 4B). HTS was used to enhance CSF influx due to its lack of effect on 
A
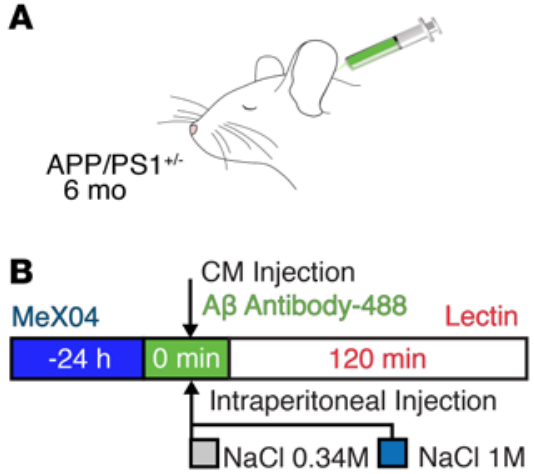

E

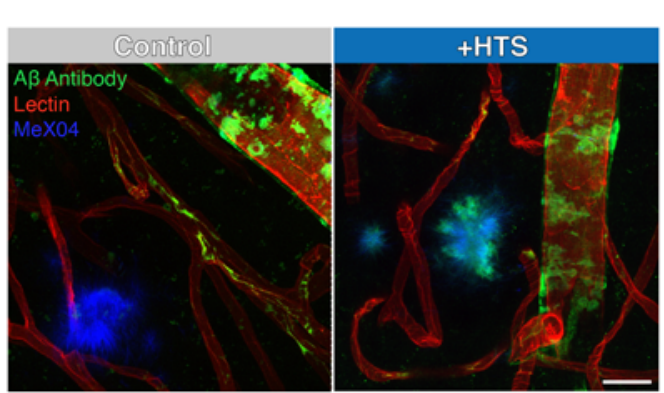

$\mathbf{F}$
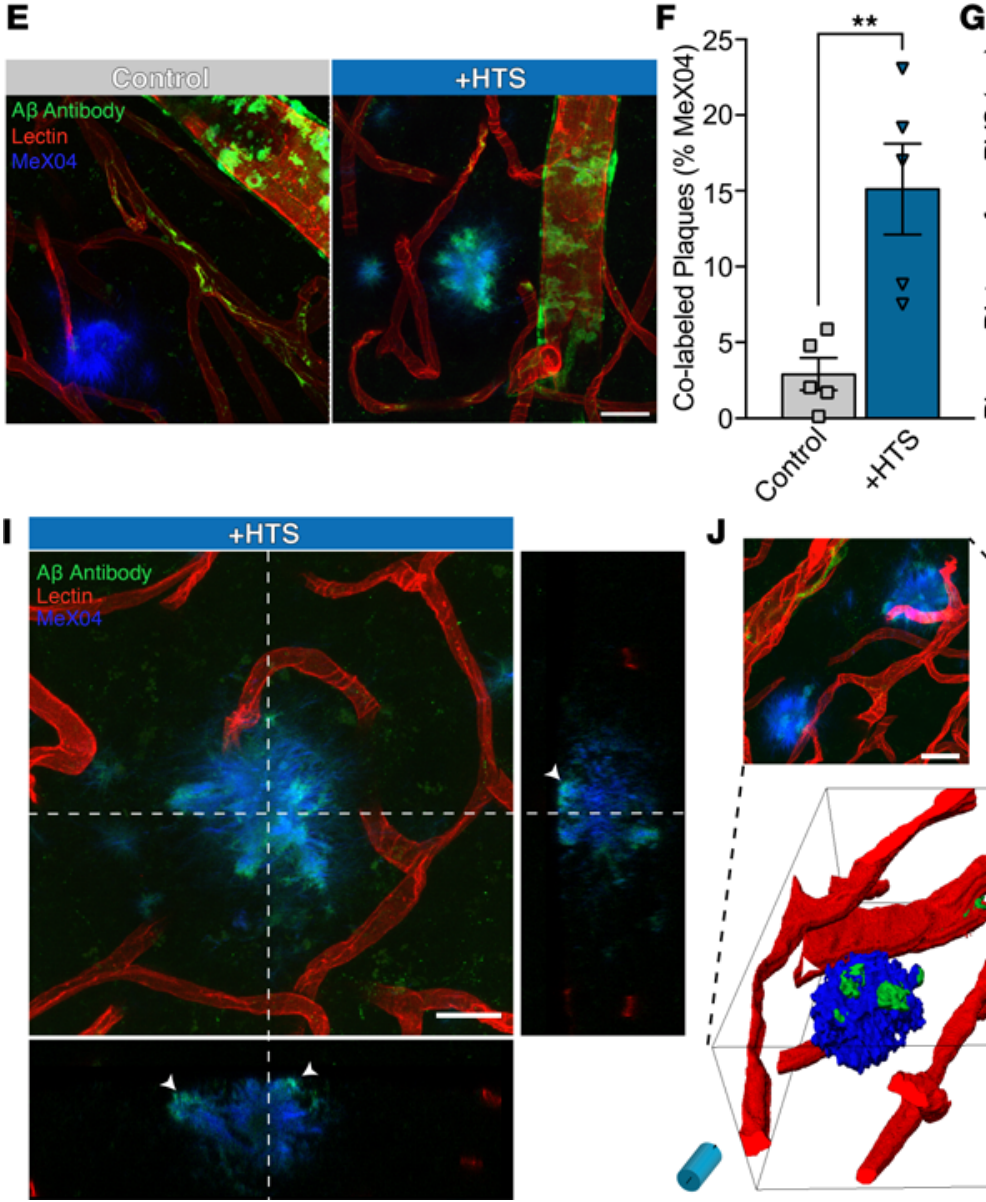

C

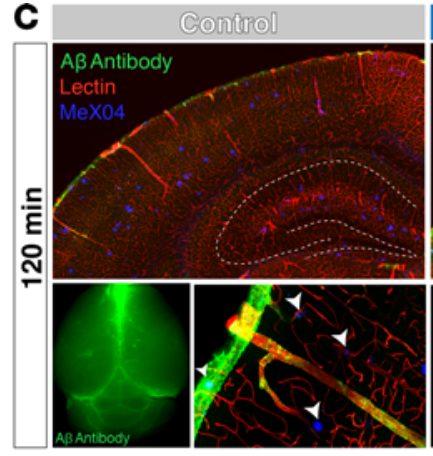

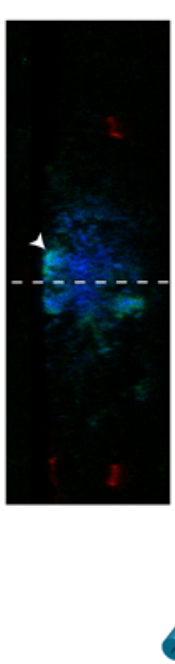

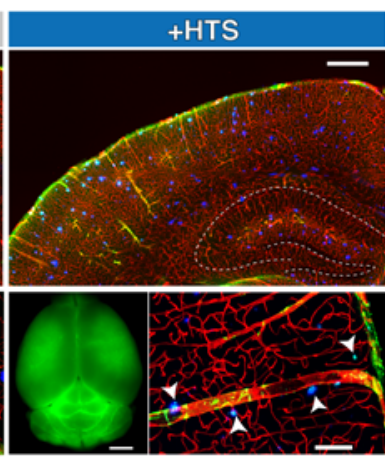

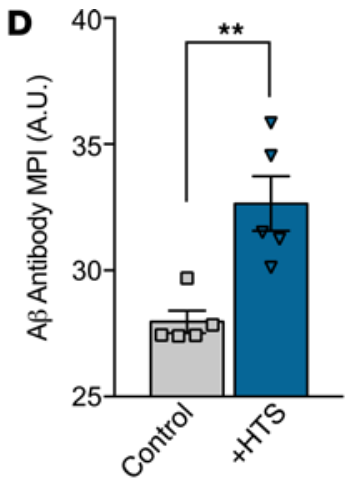

H

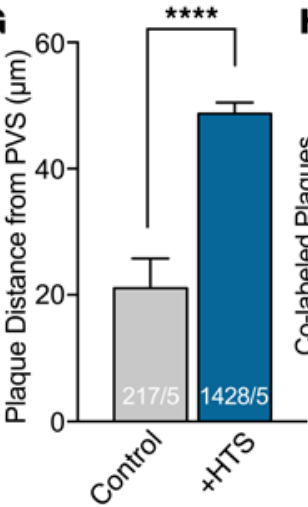

- Control $-+\mathrm{HTS}$

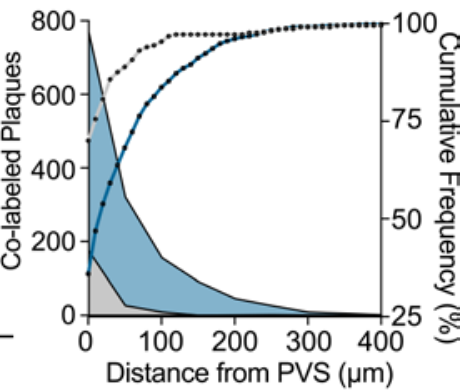

J

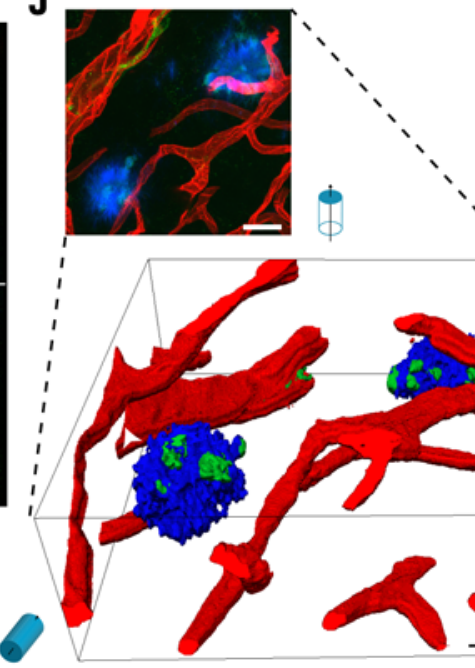

$\mathbf{K}$

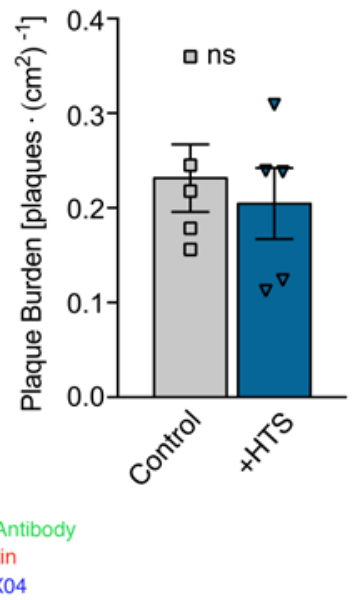

Figure 4. Plasma hypertonicity improves the delivery of an A $\beta$ antibody in 6-month-old APP/PS1 mice and enhances target engagement. (A and B) Amyloid plaques were labeled 24 hours before with methoxy-X04 (MeX04). Mice were then anesthetized, and a fluorescent anti-A $\beta$ antibody was injected intracisternally. Mice received either i.p. isotonic saline (Control) or hypertonic saline (+HTS) at the onset of the intracisternal infusion. After 120 minutes, mice were perfusion fixed with a fluorescent lectin to label the vasculature. (C) Representative ex vivo images of intact brains upon removal from the cranium (bottom left; scale bar: $2 \mathrm{~mm}$ ) and after coronal sectioning to evaluate antibody penetrance into the brain (top; scale bar: $500 \mu \mathrm{m}$ ). Confocal images of the antibody and $A \beta$ plaques (arrowheads) surrounding the perivascular spaces of penetrating arteries (bottom right; scale bar: $100 \mu \mathrm{m}$ ). (D) Quantification of ex vivo coronal section $A \beta$ antibody fluorescence MPI (mean $\pm S E M ; n=5$ mice/group; unpaired 2-tailed $t$ test; $\left.{ }^{* *} P=0.0039\right)$. (E) Representative high-magnification confocal images of perivascular $A \beta$ plaques (scale bar: $\left.20 \mu \mathrm{m}\right)$. (F) Percentage of target engagement shown by colabeling of the antibody with MeX04 ${ }^{+} \mathrm{A} \beta$ plaques (mean $\pm \mathrm{SEM} ; n=5$ mice/group; unpaired $t$ test; ${ }^{* *} P=0.005$ ). (G) Nearest neighbor analysis of the average distance of a colabeled plaque from its nearest perivascular space (PVS) in $\mu \mathrm{m}$ (mean \pm SEM; total number of colabeled plaques/number of mice in group; unpaired $t$ test; $\left.{ }^{* * *} P<0.0001\right)$. (H) Histogram and cumulative frequency plot of the number of colabeled plaques and distance from the nearest PVS. (I) Representative high-magnification confocal image with orthogonal views showing the anti-A $\beta$ antibody engaging the surface of a plaque (arrows). Scale bar: $20 \mu \mathrm{m}$. (J) Three-dimensional reconstruction of A $\beta$ plaques from an $+\mathrm{HTS}$-treated mouse showing antibody targeting and engaging plaque surface (scale bar: $20 \mu \mathrm{m}$ [both images]). (K) Plaque burden was the same between groups (mean \pm SEM; $n=5$ mice/group; unpaired $t$ test; $P=0.6165$ ). 
rCBF and only transient influence on MAP (Supplemental Figure 11, A and B). The HTS reversed the glymphatic impairment previously documented in the APP/PS1 $1^{+/-}$mice (35) and provoked significantly increased anti-A $\beta$ antibody delivery throughout the cerebrum relative to that in the isotonic controls $(P=0.0039)$. Further, the antibody appeared to gain access to the brain parenchyma via the PVSs (Figure $4, \mathrm{C}$ and $\mathrm{D})$. While the anti-A $\beta$ antibody was restricted to penetrating arterial PVSs in the control group, there was significant antibody engagement with $\mathrm{MeX04^{+ }}$ plaques in the HTS-treated mice, suggesting that plasma hypertonicity brings about redistribution of perivascular solutes to deeper interstitial sites (Figure 4C, bottom right, and Figure 4, E and F). This claim is supported by the nearest neighbor analysis, showing greater colabeled plaque distance from the nearest PVS in the +HTS group relative to that in isotonic controls (Figure 4G). Unsurprisingly, the greatest abundance of colabeled plaques occurred in the area immediately surrounding penetrating arteries, with declining frequency at greater distance from the PVS. However, nearly all colabeled plaques occurred within $100 \mu \mathrm{m}$ of the nearest periarterial space in the control group, whereas this mean separation increased to over $300 \mu \mathrm{m}$ in the HTS group (Figure $4 \mathrm{H})$. Three-dimensional reconstructions of confocal $Z$-stacks demonstrated increased antibody binding to plaque surfaces in the +HTS group, although, interestingly, there were no significant differences in plaque burden between groups (Figure 4, I-K). This is likely due to the acute setting of the experiment, being terminated after 120 minutes of antibody engagement. An important theme of future study will be to determine whether more extended periods of enhanced plaque engagement in the setting of plasma hypertonicity ultimately reduces plaque burden and rescues cognitive performance in AD mice. Present observations also extend the prior finding that genetic deletion of AQP4 in APP/PS1-transgenic mice accelerated cognitive decline and amyloid burden (54).

\section{Discussion}

Improving the delivery of drugs to the CNS is a considerable clinical challenge $(4,19,55)$, especially in the settings of experimental immunotherapy. Here, we present what we believe to be a new approach exploiting the brain-wide system of PVSs to quickly and effectively enhance delivery of therapeutics. To this end, we developed a transcranial optical imaging approach enabling noninvasive and dynamic measurements of CSF transport. We obtained brain-wide imaging of CSF tracers, in contrast to the narrow field visualized by 2-photon microscopy, while obtaining spatial and temporal resolution that is not attainable with MRI. The high frame rate acquisition is compatible with the use of front-tracking software to quantify CSF transport in the rodent brain by measuring progress of the tracer front at pixel level resolution (56). The macroscope has a large gantry to image small animals in immobilized or behaving configurations (e.g., running wheels or cognitive tests). The placement of noninvasive chronic cyanoacrylate cranial windows should eventually enable repeat imaging (57). Future work will focus on evaluating if transcranial optical imaging can be applied to intracerebroventricular or intraparenchymal tracer studies in order to evaluate clearance.

This is the first study to our knowledge describing the use of noninvasive transcranial macroscopic imaging to evaluate CSF flow patterns in rodents. We saw advective tracer inflow within the leptomeningeal PVS surrounding large cerebral arteries, which matched findings in previously validated radiometric and fluorescent ex vivo quantification methods (21). Moreover, macroscopic imaging corroborated prior findings in relation to arousal state and AQP4 expression (21). Importantly, hyperosmolar therapy with either i.p. HTS or mannitol doubled the penetration of an intracisternally delivered CSF tracer, while increasing influx speeds by about $70 \%$. This response is attributed to an increase in ISF-to-plasma efflux, causing a decrease in ICP without BBB disruption (Supplemental Figure 12). Although controlled opening of the BBB, allowing greater entry of drugs from blood to the CNS, has shown promissory results in improving drug delivery, the effect of this intervention on brain function and glymphatic clearance are yet unknown, requiring further evaluation. This intervention overcame the suppression of CSF inflow that characterizes the awake state, AQP4 depletion, and the diseased $\mathrm{AD}$ brain $(28,35)$. More specifically, plasma hypertonicity sharply improved delivery of fluorophore-conjugated $\mathrm{A} \beta$ antibody. Brain-wide distribution of the antibody resulted in significantly higher plaque engagement, with targeted plaques lying distinctly farther from the PVS despite a short CSF circulation time. Hyperosmolar therapy with intravenous hypertonic solutions is already clinically approved for the treatment of cerebral edema (49). We contend that hyperosmolar therapy should enhance immunotherapy delivery deep within the brain parenchyma. Although antibodies are large molecules, proteins as large as $2,000 \mathrm{kDa}$ can enter the brain parenchyma after intracisternal delivery $(21,26)$. Indeed, under pathological conditions such as $\mathrm{AD}$, antibodies $(>100 \mathrm{kDa})$ are transported through the PVS (27). Since this transport is 
primarily mediated by bulk flow, we expect that transport of smaller molecules is likewise enhanced. We note that exploiting hyperosmotic treatment to overcome the declining glymphatic flux in the awake state, in aging, and in disease might be combined with convection enhanced delivery strategies.

Our study shows that plasma hypertonicity can rescue impaired glymphatic function in a murine AD model, enhancing the delivery and target engagement of passive immunotherapeutics against $A \beta$. Our study also used substantially less antibody than required in previous studies, while achieving greater target engagement $(27,58)$. Future studies should aim to determine if long-term increased plaque engagement can reduce plaque burden, without increased risk of hemorrhage or other serious side effects.

\section{Methods}

Animals. For all experiments, male C57BL/6 mice, 8-12 weeks of age (Charles River), were used. Male global AQP4-knockout $\left(A q p 4^{-1-}\right)$ mice on a C57BL/6 background, between 8 and 12 weeks old, were used where indicated (21). Male 6-month-old APP/PS1 $1^{+/}$mice (The Jackson Laboratory) were used for the A $\beta$ antibody experiments.

Intracisternal injections. Mice in the KX groups were weighed and anesthetized with a mixture of ketamine (100 mg/kg, i.p.) and xylazine (10 mg/kg, i.p.). Afterward, animals were fixed in a stereotaxic frame, and the cisterna magna was surgically exposed with the help of a stereomicroscope. The cisternal space was cannulated using a 30-gauge needle attached via polyethylene tubing to a Hamilton syringe. The needle was secured with cyanoacrylate glue and tracers were infused with a syringe pump (Harvard Apparatus) depending on the experimental paradigm (see below). Mice randomized to the awake group were anesthetized with $2 \%$ isoflurane and their skulls were cemented to a customized head plate; mice were then placed in a restraint tube, after which they then underwent the same surgical procedure as described above. Tracers were allowed to circulate for 30 minutes after the injection start time and the needle was left in place for the duration of the experiment to prevent the CSF compartment from depressurizing. Core temperature $\left(37^{\circ} \mathrm{C}\right)$ and anesthetic depth were maintained throughout the experiment. At the end of 30 minutes, animals were decapitated, and the brains were processed for either fluorescent or radioisotope tracer analysis.

CSF tracers. BSA-647 (66 kDa, Invitrogen) was constituted in artificial CSF $(0.5 \% \mathrm{~m} / \mathrm{v})$ and used as a fluorescent CSF tracer. Radio-labeled ${ }^{14} \mathrm{C}$-inulin $\left(6 \mathrm{kDa}\right.$, Perkin Elmer) and ${ }^{3} \mathrm{H}$-dextran $(40 \mathrm{kDa}$, American Radiolabeled Chemicals) were dissolved in artificial CSF at a concentration of 0.1 and $10 \mu \mathrm{Ci} / \mu 1$, respectively. For APP/PS1 experiments, an Alexa Fluor 488-conjugated anti- $\beta$-amyloid antibody (clone 6E10, $1 \mathrm{mg} / \mathrm{ml}$; BioLegend, catalog 803013) was infused and allowed to circulate for 120 minutes. Fluorescent tracers and antibodies were infused in a total volume of $10 \mu \mathrm{l}$ at a rate of $2 \mu \mathrm{l} / \mathrm{min}$ or $1 \mu \mathrm{l} / \mathrm{min}$ into the cisterna magna. Radioisotope tracers were infused in a total volume of $5 \mu 1$ at $1 \mu 1 / \mathrm{min}$. A direct comparison of $2 \mu 1 / \mathrm{min}$ and $1 \mu \mathrm{l} / \mathrm{min}$ infusion rate showed no difference in BSA-647 influx on in vivo imaging $(P=0.971$, 2 -way repeated-measures ANOVA) or ex vivo coronal sections ( $P=0.939$, unpaired $t$ test)

Transcranial in vivo macroscopic imaging. For in vivo imaging, the skin covering the dorsal calvarium was incised and reflected laterally prior to cannulating the cisterna magna. The entry of CSF tracers into the brain was imaged by fluorescence macroscopy (MVX10, Olympus) using a PRIOR Lumen 1600LED light source and Flash 4.0 digital camera (Hamamatsu). The mouse fixed on the stereotaxic frame was placed on the microscope stage, and images at $\times 20$ magnification were acquired in the far-red emission channel $(647 \mathrm{~nm})$. Images $(2,048 \times 2,048$ pixel; $5.7120 \mu \mathrm{m} /$ pixel) were collected at 1 -minute intervals for 0-30 minutes following injection commencement using the MetaMorph Basic imaging software (Molecular Devices). Exposure time was held constant throughout the duration of the imaging sequence and across experimental groups.

Front tracking. To quantify the area and speed of fluorescent CSF tracer influx in the brain, we employed an algorithm recently developed in the context of advection-reaction-diffusion (56). Given a time series of a 2-dimensional concentration field, this algorithm tracks the location of the "front," which separates low-concentration and high-concentration regions. The algorithm outputs spatially and temporally resolved velocity measurements quantifying the front propagation. Influx speed is calculated by averaging over the entire group data to obtain mean front speed measurements for each group. The propagation front was identified using a threshold of 175 (on an 8-bit scale of 0 to 255); however, we noted that the results are fairly robust to different threshold choices. This same threshold was used for all images acquired during the study, which is justified since care was taken to maintain similar imaging conditions across all experiments. A fixed threshold preserves the physical meaning of the front and 
allows for quantitative comparison between populations from different experiments. More details and a copy of the code, written for MATLAB (MathWorks), are available online (56).

In vivo 2-photon imaging. A 3-mm cranial window was placed over the right parietal bone of anesthetized mice. The window was covered with agarose $\left(0.8 \%\right.$ at $\left.37^{\circ} \mathrm{C}\right)$ and sealed with a glass coverslip. Imaging was done using a resonant scanner B scope (Thorlabs) with a Chameleon Ultra II laser (Coherent) and a water-immersion $\times 20$ objective (1.0 NA, Olympus). Intravascular FITC-dextran $(2,000 \mathrm{kDa})$ was given prior to a CM tracer infusion of BSA conjugated to Texas Red (BSA-TxRd, $66 \mathrm{kDa}$ ). Z-stacks were taken over the MCA every minute from the start of the infusion for 30 minutes. To measure CSF influx, 3 circular regions of interest (ROI) were outlined on the PVS and measurements for each ROI were taken at every time point (ThorImageLS). Fluorescence intensity for all 3 ROIs were averaged and normalized to the peak fluorescence $\left(F / F_{\max }\right)$ and expressed as a percentage. Time to tracer appearance was calculated as the first time point at which fluorescence was above background signal. Orthogonal reconstructions were done using Imaris (Bitplane).

Solutions. Control mice received isosmotic saline ( $0.34 \mathrm{M} \mathrm{NaCl}$ in $\mathrm{ddH}_{2} \mathrm{O} ; 20 \mu \mathrm{l} / \mathrm{g}$, i.p.). Hyperosmolality was induced either with mannitol ( $1 \mathrm{M}$ in $0.34 \mathrm{M} \mathrm{NaCl} ; 30 \mu \mathrm{l} / \mathrm{g}$, i.p.) or HTS (1 M NaCl in ddH $\mathrm{O}_{2} ; 20$ $\mu 1 /$ g, i.p.). Thirty minutes after i.p. injection, a plasma sample was taken, and the mouse was decapitated. Plasma osmolality was measured in triplicate using a micro-osmometer (Advanced Instruments).

Brain water content measurement. Brains were dissected and immediately weighed $\left(w_{\text {wet; }} \mathrm{g}\right)$. The tissue was dried at $65^{\circ} \mathrm{C}$ until brains reached a constant weight ( 48 hours). Brains were reweighed ( $\left.w_{\text {dry }}\right)$ and the tissue water content ( $\mathrm{ml} / \mathrm{g}$ dry weight) was calculated $\left(w_{\text {wet }}-w_{\text {dry }} / w_{\text {dry }}\right)$.

Intracranial and arterial blood pressure measurements. A separate group of animals were anesthetized with a mixture of KX. Afterward, a 30-gauge needle connected to rigid polyethylene tubing filled with aCSF was inserted into the cisterna magna as described above, and an arterial catheter was placed in the femoral artery. The lines were connected to a pressure transducer and monitor (World Precision Instruments). Recordings were allowed to stabilize and then recorded continuously for 35 minutes (5 minutes baseline and 30 minutes after i.p. injection). The signals were digitized and recorded with a DigiData 1440A digitizer and AxoScope software (Axon Instruments). The intracranial and mean arterial pressure recordings were processed and analyzed using MATLAB (MathWorks).

Laser Doppler flowmetry. Relative changes in cerebral blood flow ( $\mathrm{rCBF}$ ) were measured using laser Doppler flowmetry (PF5010 Laser Doppler Perfusion Module with microtips, PR 418-1, Perimed). The tip of the fiber optic probe was fixed directly onto the exposed skull with cyanoacrylate glue. Signals were collected using a 1440A digitizer and AxoScope software (Axon Instruments). For each mouse, rCBF was recorded both 5 minutes before and 30 minutes after the administration of i.p. solutions. The rCBF recordings were processed and analyzed using MATLAB (MathWorks).

Assessment of BBB permeability. For quantification of BBB disruption, a 1\% solution of FITC-conjugated dextran $(70 \mathrm{kDa}$; MilliporeSigma) in normal saline $(4 \mathrm{ml} / \mathrm{kg}$ of body weight) was injected via the femoral vein. The dextran was allowed to circulate for 30 minutes following plasma tonicity manipulations, at which point a plasma sample was taken and the mice were perfusion fixed as described below. Positive controls received a $2 \mathrm{M}$ mannitol infusion via a catheter in the right external carotid artery $(0.64 \mathrm{ml} / \mathrm{min}$ for 30 seconds) 5 minutes after the dextran. The brains were harvested and sectioned, and FITC extravasation was imaged and quantified (see below). Plasma concentration of the FITC-dextran was calculated by diluting the plasma samples 1:4 with PBS and analyzing them in triplicate on a fluorescence microplate reader (SpectraMax M2, Molecular Devices) at $458 \mathrm{~nm}$ excitation, $538 \mathrm{~nm}$ emission, with a cutoff above $530 \mathrm{~nm}$ at room temperature $\left(24^{\circ} \mathrm{C}\right)$. Dextran concentration was estimated by comparing the relative fluorescence of the samples against a standard curve $(0.0-1.0 \mathrm{mg} / \mathrm{ml}, 0.1 \mathrm{mg} / \mathrm{ml} \mathrm{steps}$; FITC-dextran $70 \mathrm{kDa}$ in PBS) fitted with a linear regression. Blood samples with hemolysis were excluded from analysis due to interference with spectrophotometric absorbance readings.

Imaging depth analysis. The depth of fluorescence detection using macroscopic imaging is highly dependent on the power of the illumination source, excitation/emission wavelength of the fluorophore, and the exposure time. However, we attempted to estimate the depth of detection for transcranial optical imaging. For this, anesthetized mice were imaged using the macroscope while receiving an intracisternal infusion of BSA647 as before. Images were acquired every 60 seconds for a duration of 5, 10, 15, 20, 25, or 30 minutes. Mice were sacrificed immediately following the corresponding stop time; brains were harvested and drop fixed in $4 \%$ PFA overnight and then sectioned (see below). Tracer fluorescence was quantified in coronal sections to 
determine the depth of fluorescence detection (see Image analysis). In a separate set of experiments, penetration depth of imaging was calculated for a $635-\mathrm{nm}$ wavelength. To determine the optimal tracer concentration for imaging, BSA-647 was serially diluted $\left(10 \times 10^{-4}\right.$ to $1 \times 10^{-4} \mathrm{mg} / \mathrm{ml}$ by increments of 10$)$ and $10 \mu 1$ was aliquoted into a black 96-well plate. Each well was imaged on the macroscope using the same magnification and exposure time as the in vivo experiments $(100 \mathrm{~ms})$. Afterward, a capillary was loaded with $0.1 \mathrm{mg} / \mathrm{ml}$ BSA-647, sealed on both sides, and embedded in a petri dish with $4 \%$ agarose, level with the surface. Acute coronal slices of increasing thickness $(200-4,000 \mu \mathrm{m})$ were obtained from control mice and sections were placed over the dye-filled capillary for imaging. The slices were maintained in aCSF during imaging so as to preserve the optical properties of the tissue.

A $\beta$ plaque labeling. APP/PS1 mice were injected i.p. with methoxy-X04 (MeX04, Tocris, $10 \mathrm{mg} / \mathrm{kg}$, i.p.) dissolved in DMSO (10\%), propylene glycol (45\%), and PBS (45\%) 24 hours prior to cisterna magna injections, as previously described (59).

Tissue collection and processing. For fluorescent CSF tracer influx analysis, mice were decapitated after 30 minutes of the injection start and the brains drop-fixed in 4\% PFA (MilliporeSigma) overnight at $4^{\circ} \mathrm{C}$. Brains were harvested and fixed within 30 seconds of the completion of image acquisition. For assessment of $\mathrm{BBB}$ permeability, mice were transcardially perfused with ice-cold 0.1 M PBS ( $\mathrm{pH} 7.4$, MilliporeSigma) followed by $4 \%$ PFA. Brain tissue was carefully dissected away from the skull and dura and then postfixed overnight in $4 \% \mathrm{PFA}$ at $4^{\circ} \mathrm{C}$. For immunohistochemistry, mice were perfusion fixed as before, but FITC-conjugated lectin from Triticum vulgaris ( $25 \mu \mathrm{g} / \mathrm{ml}$; MilliporeSigma) was added into the ice-cold PBS solution prior to the PFA perfusion step. For the APP/PS1 experiments, mice were lectin-perfused as above but with an Alexa Fluor 647-conjugated wheat germ agglutinin lectin ( $15 \mu \mathrm{g} / \mathrm{ml}$; Invitrogen).

Immunohistochemistry. To confirm if CSF tracers entered the brain via para-arterial spaces, coronal slices were stained for AQP4 using a free-float method. Slices were blocked for 1 hour at room temperature (7\% normal donkey serum, NDS, in $0.5 \%$ Triton in PBS) and then incubated with primary rabbit anti-AQP4 (1:500; $1 \%$ NDS in $0.1 \%$ Triton/PBS, Millipore, catalog AB3594) antibody overnight at $4^{\circ} \mathrm{C}$. The sections were then incubated with a secondary Cy3-conjugated donkey anti-rabbit (1:250; Jackson ImmunoResearch, catalog 711-165-152) antibody for 2 hours at room temperature and washed. Brain sections were mounted with ProLong Gold Antifade with DAPI (Invitrogen) and allowed to dry for 24 hours before imaging.

Ex vivo fluorescence imaging. Prior to sectioning, dorsal whole brain images were acquired for CSF tracer (BSA-647) on a stereomicroscope (MVX10, Olympus) at $\times 16$ magnification. Afterward, coronal slices (100- $\mu \mathrm{m}$ thickness) were obtained using a calibrated vibratome (VT1200S, Leica). Beginning at the anterior aspect of the corpus callosum, 1 section was collected every 5 sections until a total of 6 sections had been acquired for each animal. Brain sections were mounted with ProLong Gold Antifade with DAPI (Invitrogen) and with Fluoromount-G (SouthernBiotech) for MeX04 experiments. The entry of CSF tracer into the brain was evaluated by epifluorescence macroscopy (MVX10, Olympus). Single-channel images were acquired with the MetaMorph Basic software (Molecular Devices) at low magnification ( $\times 20)$. Exposures were determined based on control brains and held constant for all groups. To better visualize tracer movement into the brain, we imaged coronal slices with a CSF tracer (BSA-647), intravital lectin (FITC), and stained for AQP4 (Cy3) on a laser scanning confocal microscope (IX81, Olympus) using FluoView (FV500, Olympus) software. Multichannel images from both left and right dorsal cortex were acquired (40$\mu \mathrm{m} Z$-stacks with 2 - $\mu \mathrm{m}$ step size at $\times 40$ magnification). In APP/PS1 mice, coronal sections were imaged at $\times 4$ magnification using a montage epifluorescence microscope (BX51 Olympus and CellSens Software) and high magnification $\times 20$ and $\times 100$ images on confocal (Leica SP8 and LASX software).

Image analysis. All images were analyzed using ImageJ software (National Institutes of Health, https:// imagej.nih.gov/ij/) (60). To measure glymphatic influx in vivo, a customized macro was developed. A ROI was defined based upon the exposed skull perimeter and overlaid on a 31-image (8-bit; 2,048 × 2,048 pixels) stack collected over the imaging session. The macro quantified mean pixel intensity for each time point ( $0-30$ minutes). Images were pseudocolored using an ImageJ lookup table (Jet) to better display pixel intensity (range, 0-255). Tracer and antibody penetration were also quantified ex vivo in coronal sections, as described previously (1). Each slice was analyzed for mean pixel intensity, and the average was computed for all 6 sections taken from one brain. For A $\beta$ plaque quantification, $\times 4$ montage coronal images were analyzed using Fiji (http://fiji. sc/) (61). Images were automatically thresholded (Yen method) on the MeX04 channel, and ROIs were generated for each plaque. Plaque burden was calculated from the mean number of plaques per $\mathrm{cm}^{2}$ from 3 coronal sections in each mouse. The thresholded MeX04 image was converted into a mask and used to calculate the 
percentage MeX04-positive area. A mask was generated for the $\mathrm{A} \beta$ antibody fluorescence following the same process, and the percentage area that was both MeX04- and A $\beta$ antibody-positive over the total MeX04-positive area was considered target engagement. The same coronal sections were used to perform a nearest neighbor analysis of the colabeled plaques and the closest PVS using Fiji and Amira (FEI). The 3-dimensional reconstruction was done using Amira (FEI) from $\times 100$ confocal $Z$-stacks ( $0.5-\mu \mathrm{m}$ step sizes, Leica SP8). To estimate the depth of fluorescence detection, mean pixel intensity for 7 ROIs, each 1-mm deep, from the dorsal convexity to the base of the brain (total $7 \mathrm{~mm}$ ) were drawn for 6 coronal sections from an individual mouse using Fiji. Background fluorescence was calculated from all the coronal slices of the 5-minute time point, and the threshold for signal was placed 2 standard deviations above background. An average for all the 1-mm ROIs from each coronal section was calculated for individual mice. Tracer was considered present when MPI from all mice in the group was higher than the threshold. BBB permeability to FITC-dextran was quantified as percentage area in 6 coronal sections for each mouse using a thresholding approach. The threshold was established using Fiji on all coronal sections from the positive control group and computing an average for all slices. The average threshold level was then applied to all the sections from the experimental groups.

Radioisotope influx. To evaluate solute influx into the brain, radiolabeled tracers ${ }^{3} \mathrm{H}$-dextran $(50 \mu \mathrm{Ci})$ and ${ }^{14} \mathrm{C}$-inulin $(0.5 \mu \mathrm{Ci})$ were injected into the cisterna magna as described above. After 30 minutes, animals were rapidly decapitated, the skull and dura were removed, and the brain was harvested. Brain radioactivity was normalized to the total radioactivity detected in a $5-\mu 1$ aliquot put directly into a scintillation vial immediately before intracisternal injection. All brain tissue was weighed and solubilized in $0.5 \mathrm{ml}$ tissue solubilizer (Solvable, PerkinElmer) overnight. Upon solubilization, $5 \mathrm{ml}$ scintillation cocktail was added (Ultima Gold, PerkinElmer). The injectate controls were treated in the same way as the tissue samples. All samples were analyzed by liquid scintillation spectrometry using a scintillation counter (LS 6500 Multipurpose Scintillation Counter, Beckman Coulter). The radioactivity (disintegrations per minute) remaining in the brain after injection (percentage of injected dose [\%ID]) was determined as $R_{b} / R_{i} \times 100$, where $R_{b}$ is the radioactivity remaining in the brain at the end of the experiment and $\mathrm{R}_{\mathrm{i}}$ is the radioactivity in the injectate controls for each experiment. Influx percentage was deduced as \%ID. Dextran and inulin are inert, polar molecules that are not actively transported within the CNS, and due to their difference in molecular weight, they are ideal tracers for evaluating the presence of bulk flow.

Statistics. All statistical testing was performed on GraphPad Prism 7. Tests were chosen based on the data set being analyzed and are reported in the figure legends. They include repeated-measures 2-way ANOVA, Sidak's multiple comparisons test; 1-way ANOVA, Tukey's multiple comparisons test; and unpaired 2-tailed $t$ test. All statistical testing was 2 tailed, and exact $P$ values were calculated at a 0.05 level of significance. All values are expressed as the mean \pm SEM, unless otherwise stated.

Study approval. All experiments adhered to the laws of the United States and the regulations of the Department of Agriculture and were performed according to guidelines from the National Institutes of Health (Guide for the Care and Use of Laboratory Animals, National Academies Press, 2011). Experiments were approved by the University Committee on Animal Resources of the University of Rochester (protocol 2011-023), and an effort was made to minimize the number of animals used.

\section{Author contributions}

BAP, HM, and MN conceived the project and designed all experiments. BAP, HM, GEO, AMS, HMK, AC, KYD, and TD performed all experiments. DHK and TDN developed the front-tracking software, and JT and TDN performed the front-tracking analysis. IL assisted with confocal imaging. BAP, HM, JT, DHK, and MN wrote and edited the manuscript.

\section{Acknowledgments}

This work was supported by the National Institutes of Health (R01NS100366 to MN; RF1AG057575 to $\mathrm{MN}$ and DHK). This work was also supported by the Office of the Assistant Secretary of Defense for Health Affairs under the Peer Reviewed Alzheimer's Research Program, through the award W81XWH-16-1-0555. Opinions, interpretations, conclusions, and recommendations are those of the authors and are not necessarily endorsed by the Department of Defense. TDN was supported by the Department of Defense National Defense Science \& Engineering Graduate Fellowship program. We would like to thank Dan Xue for assistance with illustrations; Wei Song, Weiguo Peng, and Nanhong Lou for expert surgical assistance; and Yunpeng Pang for assistance with immunofluorescent staining. 
Address correspondence to: Maiken Nedergaard, Center for Translational Neuromedicine, Department of Neurosurgery, University of Rochester Medical Center, 601 Elmwood Avenue, Box 645, Rochester, New York 14642, USA. Phone: 585.273.2868; Email: maiken_nedergaard@urmc.rochester.edu.

1. Sevigny J, et al. The antibody aducanumab reduces A $\beta$ plaques in Alzheimer's disease. Nature. 2016;537(7618):50-56

2. Schenk DB, et al. First-in-human assessment of PRX002, an anti- $\alpha$-synuclein monoclonal antibody, in healthy volunteers. Mov Disord. 2017;32(2):211-218

3. Gros-Louis F, Soucy G, Larivière R, Julien JP. Intracerebroventricular infusion of monoclonal antibody or its derived Fab fragment against misfolded forms of SOD1 mutant delays mortality in a mouse model of ALS. J Neurochem. 2010;113(5):1188-1199.

4. Gallardo G, Holtzman DM. Antibody therapeutics targeting A $\beta$ and Tau. Cold Spring Harb Perspect Med. 2017;7:a024331.

5. Sampson JH, Maus MV, June CH. Immunotherapy for brain tumors. J Clin Oncol. 2017;35(21):2450-2456.

6. Selkoe DJ, Hardy J. The amyloid hypothesis of Alzheimer's disease at 25 years. EMBO Mol Med. 2016;8(6):595-608

7. Klyubin I, et al. Amyloid beta protein immunotherapy neutralizes Abeta oligomers that disrupt synaptic plasticity in vivo. Nat Med. 2005;11(5):556-561.

8. Salloway S, et al. Two phase 3 trials of bapineuzumab in mild-to-moderate Alzheimer's disease. N Engl J Med. 2014;370(4):322-333.

9. Doody RS, et al. Phase 3 trials of solanezumab for mild-to-moderate Alzheimer's disease. N Engl J Med. 2014;370(4):311-321.

10. Honig LS, et al. Trial of solanezumab for mild dementia due to Alzheimer's disease. N Engl J Med. 2018;378(4):321-330

11. Vandenberghe R, et al. Bapineuzumab for mild to moderate Alzheimer's disease in two global, randomized, phase 3 trials. $A l z-$ heimers Res Ther. 2016;8(1):18.

12. Brody DL, Holtzman DM. Active and passive immunotherapy for neurodegenerative disorders. Annu Rev Neurosci. 2008;31:175-193.

13. Calias P, Banks WA, Begley D, Scarpa M, Dickson P. Intrathecal delivery of protein therapeutics to the brain: a critical reassessment. Pharmacol Ther. 2014;144(2):114-122.

14. Cohen-Pfeffer JL, et al. intracerebroventricular delivery as a safe, long-term route of drug administration. Pediatr Neurol. 2017;67:23-35.

15. Prins ND, Scheltens P. Treating Alzheimer's disease with monoclonal antibodies: current status and outlook for the future. Alzheimers Res Ther. 2013;5(6):56.

16. Banks WA, Terrell B, Farr SA, Robinson SM, Nonaka N, Morley JE. Passage of amyloid beta protein antibody across the blood-brain barrier in a mouse model of Alzheimer's disease. Peptides. 2002;23(12):2223-2226.

17. Banks WA, Farr SA, Morley JE, Wolf KM, Geylis V, Steinitz M. Anti-amyloid beta protein antibody passage across the bloodbrain barrier in the SAMP8 mouse model of Alzheimer's disease: an age-related selective uptake with reversal of learning impairment. Exp Neurol. 2007;206(2):248-256.

18. Salloway S, Sperling R, Brashear HR. Phase 3 trials of solanezumab and bapineuzumab for Alzheimer's disease. $N$ Engl J Med. 2014;370(15):1460.

19. Thakker DR, et al. Intracerebroventricular amyloid-beta antibodies reduce cerebral amyloid angiopathy and associated micro-hemorrhages in aged Tg2576 mice. Proc Natl Acad Sci USA. 2009;106(11):4501-4506.

20. Sperling RA, et al. Amyloid-related imaging abnormalities in amyloid-modifying therapeutic trials: recommendations from the Alzheimer's Association Research Roundtable Workgroup. Alzheimers Dement. 2011;7(4):367-385.

21. Iliff JJ, et al. A paravascular pathway facilitates CSF flow through the brain parenchyma and the clearance of interstitial solutes, including amyloid $\beta$. Sci Transl Med. 2012;4(147):147ra111.

22. Iliff JJ, et al. Brain-wide pathway for waste clearance captured by contrast-enhanced MRI. J Clin Invest. 2013;123(3):1299-1309.

23. Jessen NA, Munk AS, Lundgaard I, Nedergaard M. The glymphatic system: a beginner's guide. Neurochem Res. 2015;40(12):2583-2599.

24. Rennels ML, Gregory TF, Blaumanis OR, Fujimoto K, Grady PA. Evidence for a 'paravascular' fluid circulation in the mammalian central nervous system, provided by the rapid distribution of tracer protein throughout the brain from the subarachnoid space. Brain Res. 1985;326(1):47-63.

25. Rennels ML, Blaumanis OR, Grady PA. Rapid solute transport throughout the brain via paravascular fluid pathways. Adv Neurol. 1990;52:431-439.

26. Wolak DJ, Pizzo ME, Thorne RG. Probing the extracellular diffusion of antibodies in brain using in vivo integrative optical imaging and ex vivo fluorescence imaging. J Control Release. 2015;197:78-86.

27. Pizzo ME, et al. Intrathecal antibody distribution in the rat brain: surface diffusion, perivascular transport and osmotic enhancement of delivery. J Physiol (Lond). 2018;596(3):445-475

28. Xie L, et al. Sleep drives metabolite clearance from the adult brain. Science. 2013;342(6156):373-377.

29. Kress BT, et al. Impairment of paravascular clearance pathways in the aging brain. Ann Neurol. 2014;76(6):845-861.

30. Iliff JJ, et al. Cerebral arterial pulsation drives paravascular CSF-interstitial fluid exchange in the murine brain. $J$ Neurosci. 2013;33(46):18190-18199.

31. Lee H, et al. The effect of body posture on brain glymphatic transport. J Neurosci. 2015;35(31):11034-11044.

32. Pullen RG, DePasquale M, Cserr HF. Bulk flow of cerebrospinal fluid into brain in response to acute hyperosmolality. Am J Physiol. 1987;253(3 Pt 2):F538-F545.

33. Cserr HF, DePasquale M, Patlak CS. Volume regulatory influx of electrolytes from plasma to brain during acute hyperosmolality. Am J Physiol. 1987;253(3 Pt 2):F530-F537.

34. Cserr HF, DePasquale M, Nicholson C, Patlak CS, Pettigrew KD, Rice ME. Extracellular volume decreases while cell volume is maintained by ion uptake in rat brain during acute hypernatremia. J Physiol (Lond). 1991;442:277-295.

35. Peng W, et al. Suppression of glymphatic fluid transport in a mouse model of Alzheimer's disease. Neurobiol Dis. 2016;93:215-225.

36. Yang L, et al. Evaluating glymphatic pathway function utilizing clinically relevant intrathecal infusion of CSF tracer. J Transl 
Med. 2013;11:107.

37. Mestre $\mathrm{H}$ et al. Aquaporin-4 dependent glymphatic solute transport in rodent brain. bioRxiv. https://doi.org/10.1101/216499. Published November 9, 2017. Accessed September 17, 2018.

38. Lundgaard I, et al. Glymphatic clearance controls state-dependent changes in brain lactate concentration. J Cereb Blood Flow Metab. 2017;37(6):2112-2124.

39. Achariyar TM, et al. Glymphatic distribution of CSF-derived apoE into brain is isoform specific and suppressed during sleep deprivation. Mol Neurodegener. 2016;11(1):74.

40. Smith AJ, Yao X, Dix JA, Jin BJ, Verkman AS. Test of the 'glymphatic' hypothesis demonstrates diffusive and aquaporin-4-independent solute transport in rodent brain parenchyma. Elife. 2017;6:e27679.

41. Murlidharan G, Crowther A, Reardon RA, Song J, Asokan A. Glymphatic fluid transport controls paravascular clearance of AAV vectors from the brain. JCI Insight. 2016;1(14):e88034.

42. Jiang Q, et al. Impairment of the glymphatic system after diabetes. J Cereb Blood Flow Metab. 2017;37(4):1326-1337.

43. Rangroo Thrane V, et al. Paravascular microcirculation facilitates rapid lipid transport and astrocyte signaling in the brain. Sci Rep. 2013;3:2582.

44. Lundgaard I, et al. Direct neuronal glucose uptake heralds activity-dependent increases in cerebral metabolism. Nat Commun. 2015;6:6807.

45. Venkat $\mathrm{P}$, et al. White matter damage and glymphatic dysfunction in a model of vascular dementia in rats with no prior vascular pathologies. Neurobiol Aging. 2017;50:96-106.

46. Benveniste $\mathrm{H}$, et al. Anesthesia with dexmedetomidine and low-dose isoflurane increases solute transport via the glymphatic pathway in rat brain when compared with high-dose isoflurane. Anesthesiology. 2017;127(6):976-988.

47. Gaberel T, et al. Impaired glymphatic perfusion after strokes revealed by contrast-enhanced MRI: a new target for fibrinolysis? Stroke. 2014;45(10):3092-3096

48. Ringstad G, Vatnehol SAS, Eide PK. Glymphatic MRI in idiopathic normal pressure hydrocephalus. Brain. 2017;140(10):2691-2705.

49. Brain Trauma Foundation, et al. Guidelines for the management of severe traumatic brain injury. II. Hyperosmolar therapy. J Neurotrauma. 2007;24 Supp1 1:S14-S20.

50. Ma Q, Ineichen BV, Detmar M, Proulx ST. Outflow of cerebrospinal fluid is predominantly through lymphatic vessels and is reduced in aged mice. Nat Commun. 2017;8(1):1434.

51. Bedussi B, et al. Paravascular channels, cisterns, and the subarachnoid space in the rat brain: A single compartment with preferential pathways. J Cereb Blood Flow Metab. 2017;37(4):1374-1385.

52. Muizelaar JP, Lutz HA, Becker DP. Effect of mannitol on ICP and CBF and correlation with pressure autoregulation in severely head-injured patients. J Neurosurg. 1984;61(4):700-706.

53. Domaingue CM, Nye DH. Hypotensive effect of mannitol administered rapidly. Anaesth Intensive Care. 1985;13(2):134-136.

54. Xu Z, et al. Deletion of aquaporin-4 in APP/PS1 mice exacerbates brain A $\beta$ accumulation and memory deficits. Mol Neurodegener. 2015;10:58.

55. Golde TE. Open questions for Alzheimer's disease immunotherapy. Alzheimers Res Ther. 2014;6(1):3.

56. Nevins TD, Kelley DH. Front tracking for quantifying advection-reaction-diffusion. Chaos. 2017;27(4):043105.

57. Silasi G, Xiao D, Vanni MP, Chen AC, Murphy TH. Intact skull chronic windows for mesoscopic wide-field imaging in awake mice. J Neurosci Methods. 2016;267:141-149.

58. Demattos RB, et al. A plaque-specific antibody clears existing $\beta$-amyloid plaques in Alzheimer's disease mice. Neuron. 2012;76(5):908-920.

59. Klunk WE, et al. Imaging Abeta plaques in living transgenic mice with multiphoton microscopy and methoxy-X04, a systemically administered Congo red derivative. J Neuropathol Exp Neurol. 2002;61(9):797-805.

60. Schneider CA, Rasband WS, Eliceiri KW. NIH Image to ImageJ: 25 years of image analysis. Nat Methods. 2012;9(7):671-675.

61. Schindelin J, et al. Fiji: an open-source platform for biological-image analysis. Nat Methods. 2012;9(7):676-682. 